\section{Preservación de datos de investigación: estrategias prácticas}

\author{
Antonia Ferrer-Sapena \\ Universitat Politècnica de València \\ anfersa@upv.es
}

\section{Enrique A. Sánchez-Pérez \\ Universitat Politècnica de València easancpe@mat.upv.es}

\section{Fernanda Peset}

Universitat Politècnica de València mpesetm@upv.es

Ferrer-Sapena, Antonia; Sánchez-Pérez, Enrique A.; Peset, Fernanda (2018). "Preservación de datos de investigación: estrategias prácticas". Anuario ThinkEPI, v. 12, pp. 335-336.

https://doi.org/10.3145/thinkepi.2018.53

Publicado en Blok de BiD el 12 de julio de 2017

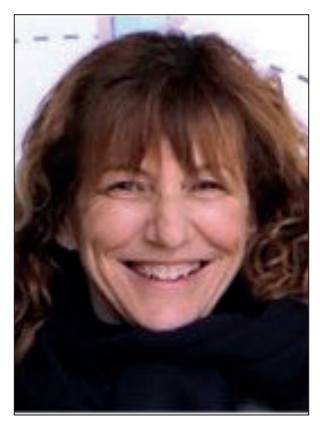

\section{Introducción}

Este es el primer volumen de una serie llamada Curating research data, en el que se exploran las razones por las que existen servicios de preservación de los datos. Consta de tres partes y una introducción.

Lisa R. Johnston resume

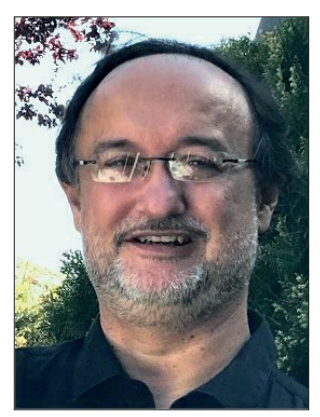

los principales apartados del libro. Johnston ha sido bibliotecaria de los servicios de investigación y codirectora del repositorio institucional de la University of Minnesota, capacitación que le permite señalar los desafíos a los que se enfrentan los profesionales encargados del tratamiento de los datos.

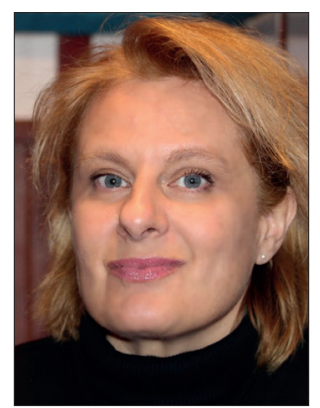

Los considera los primeros usuarios, por lo que son quienes desde el primer momento deben identificar los problemas que puede tener su reutilización. En esta introducción, además, se define la terminología que se utiliza así como los motivos por los cuales se desarrollan los servicios de conservación, los requisitos que deben tener y las políticas para su intercambio.

\section{Preservación}

Se exponen políticas existentes para el intercambio de datos y se señalan algunos de los trabajos de colaboración puestos en marcha. Se incide en cuestiones ya conocidas, como la influencia de los incentivos -positivos o negativos- académicos y de los organismos de financiación para abrir datos, y cómo han contribuido a que se extienda la colaboración en áreas en las que hasta el momento era escasa. En esta primera parte, destaca la recopilación de políticas de fomento para compartir datos tanto a nivel nacional como internacional en varios países. Junto a los mandatos de los países, también se aborda la política que están siguiendo las revistas para el depósito de datos, señalando las publicaciones y estudios que se han hecho. Obviamente, toma como referencia los estudios de Heather A. Piwowar. Esta primera parte del libro se cierra con estudios de casos de éxito y normas para la reutilización.

\section{Mantenimiento de los servicios sobre datos de investigación}

Algunos de los factores clave que identifica son los costos, las estrategias de marketing y dónde deben focalizar el esfuerzo los profesionales que se enfrentan al reto de poner en marcha un repositorio de datos. Jon Wheeler muestra cómo las bibliotecas académicas han ido tomando un papel cada vez más significativo en la prestación de servicios tanto básicos como avanzados. Plantea, por ejemplo, cómo ofrecer plataformas para la publicación de datos junto con el repositorio. Establece de qué manera estos repositorios

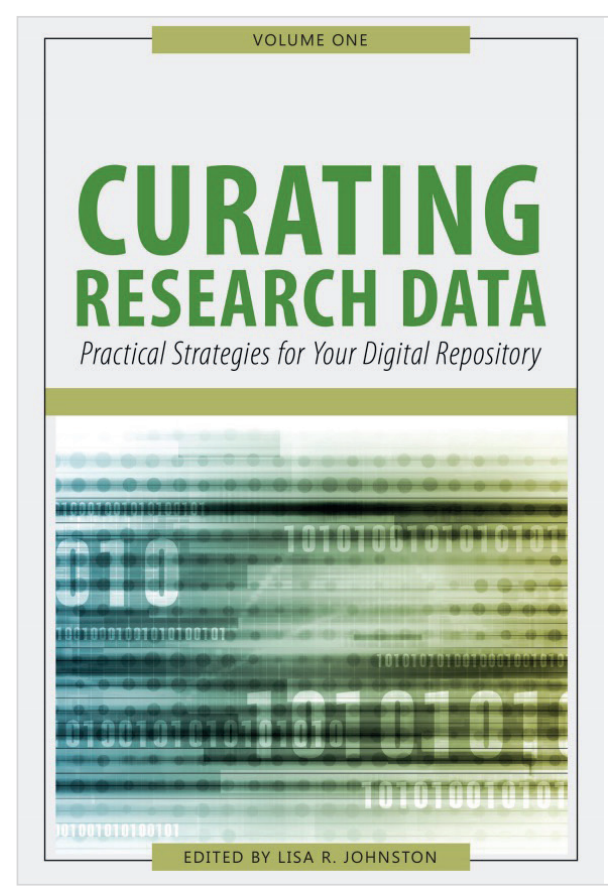


pueden ser complementarios a los repositorios temáticos, lo que ilustra con ejemplos, presentando modelos de financiación que pueden hacerlos sostenibles a largo plazo.

En el capítulo 9, Katherine J. Gerwig, de la Metropolitan State University, muestra los métodos de marketing y difusión empleados por más de una docena de instituciones académicas. Ello puede ser útil para generar nuevas ideas a quienes comiencen con políticas de difusión en los repositorios de datos.

\section{"Documentar los detalles no suele ser del agrado del científico, que lo considera una tarea meramente técnica y no ligada a sus intereses investigadores"}

\section{Ciclo de vida de los datos}

Trata cuestiones prácticas y éticas que los profesionales deben tener en cuenta cuando preparan los datos para la preservación. Si se conservan para su posterior reutilización, el usuario final debe tener disponibles todos los metadatos: cómo se diseñaron y construyeron los datos; cuándo, dónde y cómo se recopilaron; y una minuciosa descripción de su procesamiento. Documentar todos estos detalles no suele ser del agrado del científico que lo considera una tarea meramente técnica y no ligada a sus intereses investigadores. De forma adicional, en ocasiones tampoco son partidarios de una transparencia extrema de los datos, ya que esto los hace más vulnerables a los ataques y a la apropiación de trabajos. Ante estos problemas, se plantea que el curador de los datos puede adoptar un rol que contribuya a eliminar estas suspicacias, promoviendo la reutilización ética y adecuada de los mismos.

Un punto poco tratado es el del capítulo 12 , crucial para la preservación: ¿qué ocurre cuando los datos ya son obsoletos? También se presentan problemas a la hora de recuperar datos de servicios que ya han desaparecido, u otros ligados a los datos huérfanos. En estos casos se les atribuye a los curadores la labor de aplicar metadatos (en algunos casos) para que puedan ser reutilizados.

Finaliza este volumen con una reflexión sobre dos aspectos:

- las colecciones digitales de datos como catalizadores del progreso y su contribución a la democratización de la educación y la ciencia;

- contribución de los datos abiertos a la mejora del desarrollo económico y social, y si pueden llegar a ser económicamente rentables.
Johnston, Lisa R. (ed.) Curating research data. Chicago: Association of College and Research Libraries, 2017-. Volume one: practical strategies for your digital repository. $285 \mathrm{pp}$.

http://www.ala.org/acrl/sites/ala.org.acrl/files/ content/publications/booksanddigitalresources/ digital/9780838988596_crd_v1_OA.pdf

Nota original completa en Blok de BiD:

http://www.ub.edu/blokdebid/es/node/803

***

\section{Acceso digital a las monografías académicas: una tarea todavía llena de dificultades}

\section{Ángel Borrego}

Universitat de Barcelona

Facultat de Biblioteconomia i Documentació borrego@ub.edu

Borrego, Ángel (2018). "Acceso digital a las monografías académicas: una tarea todavía llena de dificultades". Anuario ThinkEPI, v. 12, pp. 336-338.

https://doi.org/10.3145/thinkepi.2018.54

Publicado en Blok de BiD el 19 de julio de 2017

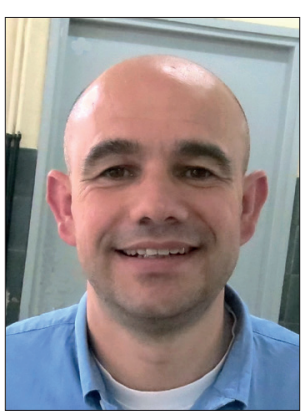

\section{Estudio piloto}

En 2014, Jisc, Research Libraries UK (RLUK) y Society for Colleges, National and University Libraries (Sconul) llevaron a cabo un proyecto que, bajo el título National monograph strate$g y$, analizaba los principales problemas que planteaba el acceso a monografías académicas en bibliotecas universitarias y de investigación. Las conclusiones de aquel proyecto incluían siete recomendaciones, dos de las cuales hacían referencia a la necesidad de una estrategia de digitalización y un modelo de licencia nacional para el acceso a las monografías que no estén disponibles en formato digital.

El informe que ahora reseñamos presenta las conclusiones de un estudio piloto que ha trabajado en el desarrollo de aquellas dos recomendaciones. La aproximación utilizada consistió en pedir a una decena de bibliotecas universitarias 